\title{
THE EXPONENTIAL SPECTRUM IN BANACH ALGEBRAS
}

\author{
ROBIN HARTE
}

\begin{abstract}
ABSTRACr. The exponential spectrum of a Banach algebra element is introduced, and used to obtain a spectral permanence result for quotient algebras.
\end{abstract}

1. Suppose $A$ and $B$ are Banach algebras, in the sense of complex Banach algebras with identity $1 \neq 0$, and suppose $T: A \rightarrow B$ is a homomorphism, in the sense of a bounded linear homomorphism which preserves identity: then there is inclusion $T\left(A^{-1}\right) \subseteq B^{-1}$, where $A^{-1}=\left\{a \in A: \exists a^{\prime} \in A, a^{\prime} a=a a^{\prime}\right.$ $=1\}$ denotes the group of invertible elements of $A$, and hence for each $a \in A$

$$
\sigma_{B}(T a) \subseteq \bigcap_{T a^{\prime}=0} \sigma_{A}\left(a+a^{\prime}\right),
$$

where $\sigma_{A}(a)=\left\{s \in \mathbf{C}: a-s \notin A^{-1}\right\}$ is the spectrum of $a$ in $A$. We shall show in this note that if the homomorphism $T$ has closed range then for each $a \in A$

$$
\bigcap_{a^{\prime}=0} \sigma_{A}\left(a+a^{\prime}\right) \subseteq \eta \sigma_{B}(T a),
$$

where $\eta K$ denotes the connected hull of a compact subset $K \subseteq \mathbf{C}$ :

$$
\mathbf{C} \backslash \eta K \text { is the unbounded component of } \mathbf{C} \backslash K .
$$

The derivation of (1.2) is familiar if $T$ is isometric, and the argument extends to the case in which $T$ is "bounded below". We need an auxiliary spectrum: if $\tau_{A}(a)$ denotes the "singular spectrum" of $a$ in $A$,

$$
\tau_{A}(a)=\{s \in \mathbf{C}: a-s \text { is a topological zero-divisor in } A\},
$$

then it is familiar that

$$
\partial \sigma_{A}(a) \subseteq \tau_{A}(a) \subseteq \sigma_{A}(a),
$$

where $\partial K$ denotes the topological boundary of $K$ in $\mathbf{C}$. If the homomorphism $T: A \rightarrow B$ is bounded below, in the sense that $\inf _{\|a\| \geqslant 1}\|T a\|>0$, then at once

Received by the editors November 18, 1974 and, in revised form, August 1, 1975.

AMS (MOS) subject classifications (1970). Primary 46H05, 47A10; Secondary 47B30.

Key words and phrases. Generalized exponentials in a Banach algebra, boundary of the spectrum, connected hull of a compact set. 


$$
\tau_{A}(a) \subseteq \tau_{B}(T a) .
$$

Finally if $K_{1}$ and $K_{2}$ are compact subsets of $\mathbf{C}$ it is an easy exercise that

$$
\partial K_{1} \subseteq K_{2} \Rightarrow K_{1} \subseteq \eta K_{2} .
$$

Putting $K_{1}=\sigma_{A}(a)$ and $K_{2}=\sigma_{B}(T a)$ gives (1.2).

2. In some sense dual to the nontopological zero-divisors of $A$ are the generalized exponentials

$$
\operatorname{Exp}(A)=\left\{e^{c_{1}} e^{c_{2}} \cdots e^{c_{k}}: k \in \mathbf{N} ; c_{1}, c_{2}, \ldots, c_{k} \in A\right\},
$$

which form the connected component of the identity 1 in the topological group $A^{-1}$ [2, Chapter 2], [5, §4.6].

Definition 1. If $a \in A$ then the exponential spectrum of $a$ in $A$ is the set

$$
\varepsilon_{A}(a)=\{s \in \mathbf{C}: a-s \notin \operatorname{Exp}(A)\} .
$$

THEOREM 1. If $a \in A$ then $\varepsilon_{A}(a)$ is compact and nonempty and satisfies

$$
\partial \varepsilon_{A}(a) \subseteq \tau_{A}(a) \subseteq \sigma_{A}(a) \subseteq \varepsilon_{A}(a) \subseteq \eta \sigma_{A}(a) .
$$

Proof. Since $\operatorname{Exp}(A)$ is open it is obvious that $\varepsilon_{A}(a)$ is closed, and the boundedness and nonemptiness will follow from (2.3). Obviously $\sigma_{A}(a)$ $\subseteq \varepsilon_{A}(a)$, since $\operatorname{Exp}(A) \subseteq A^{-1}$. We claim that $\partial \varepsilon_{A}(a) \subseteq \tau_{A}(a)$ : for suppose that $s \in \varepsilon_{A}(a)$ is the limit of a sequence $\left(s_{n}\right)$ of points outside $\varepsilon_{A}(a)$. The elements $a-s_{n}$ will, in particular, be invertible, and we claim that

$$
\left|s_{n}-s\right|\left\|\left(a-s_{n}\right)^{-1}\right\| \geqslant 1 \text { for all } n .
$$

If not, then for some $n$ the element

$$
(a-s)\left(a-s_{n}\right)^{-1}=1-\left(s-s_{n}\right)\left(a-s_{n}\right)^{-1}
$$

will be in $\operatorname{Exp}(A)$, hence also the element $a-s$, contradicting $s \in \varepsilon_{A}(a)$. The proof now follows the derivation of (1.5): with

$$
b_{n}=\left(a-s_{n}\right)^{-1} /\left\|\left(a-s_{n}\right)^{-1}\right\|
$$

we have $\left\|b_{n}\right\|=1$ and $\left\|(a-s) b_{n}\right\| \leqslant 2\left|s_{n}-s\right| \rightarrow 0$, giving $s \in \tau_{A}(a)$. Using (1.7), the proof of (2.3) is complete.

If $T: A \rightarrow B$ is a homomorphism then there is inclusion $T(\operatorname{Exp}(A))$ $\subseteq \operatorname{Exp}(B)$ and hence, if $a \in A$,

$$
\varepsilon_{B}(T a) \subseteq \underset{T a^{\prime}=0}{\bigcap} \varepsilon_{A}\left(a+a^{\prime}\right) .
$$

THeorem 2. If the homomorphism $T: A \rightarrow B$ is onto then $T(\operatorname{Exp}(A))$ $=\operatorname{Exp}(B)$ and hence for each $a \in A$

$$
\bigcap_{T a^{\prime}=0} \varepsilon_{A}\left(a+a^{\prime}\right)=\varepsilon_{B}(T a) .
$$


Proof. If $b \in B$ is in $\operatorname{Exp}(B)$, so that $b=e^{d_{1}} e^{d_{2}} \cdots e^{d_{k}}$ for some $k$ $\in \mathbf{N}$ and $d_{j} \in B$ then $d_{j}=T\left(c_{j}\right)$ for some $c_{j} \in A$ and hence $b=T a$ with $a=e^{c_{1}} e^{c_{2}} \cdots e^{c_{k}} \in \operatorname{Exp}(A)$. Now for (2.5) suppose that $s \in \mathbf{C}$ is not in $\varepsilon_{B}(T a)$, so that $T a-s=T a^{\prime \prime}$ for some $a^{\prime \prime} \in \operatorname{Exp}(A)$ : then $a^{\prime}=a^{\prime \prime}-a+s$ gives $T a^{\prime}=0$ and $a+a^{\prime}-s \in \operatorname{Exp}(A)$.

The derivation of (1.2) for an onto homomorphism $T: A \rightarrow B$ is effected by (2.3) and (2.5).

THEOREM 3. If the homomorphism $T: A \rightarrow B$ has closed range then (1.2) holds.

Proof. If we factorize $T: A \rightarrow A / J \stackrel{T^{\wedge}}{\longrightarrow} B$, where $J=T^{-1}(0)$, then the natural mapping $a \rightarrow a+J$ is onto, while $T^{\hat{1}}$ is one-one with closed range, hence bounded below. Now

$$
\bigcap_{T a^{\prime}=0} \sigma_{A}\left(a+a^{\prime}\right) \subseteq \eta \sigma_{A / J}(a+J) \subseteq \eta \eta \sigma_{B}(T a)=\eta \sigma_{B}(T a) .
$$

The implication (1.7) is not reversible for compact subsets of $\mathbf{C}$ : this leaves us with the following

Problem 1. If $T: A \rightarrow B$ has closed range and $a \in A$, is there inclusion

$$
\partial\left(\bigcap_{T a^{\prime}=0} \sigma_{A}\left(a+a^{\prime}\right)\right) \subseteq \sigma_{B}(T a) ?
$$

The assumption that $T$ has closed range cannot be dropped from Theorem 3. The simplest example uses disc algebras: if $t>0$ write $t \mathbf{D}=\{s \in C:|s|$ $\leqslant t\}$ and denote by $\mathscr{Q}(t \mathbf{D})$ the algebra of functions continuous on $t \mathbf{D}$ and analytic on its interior.

EXAMPle 1. If $T: A \rightarrow B$ is the restriction mapping from $A=\mathbb{Q}(\mathbf{D})$ into $B=\mathscr{Q}\left(\frac{1}{2} \mathbf{D}\right)$ and $a=z \in A$ then (1.2) fails.

For $\sigma_{A}(a)=\cap_{T a^{\prime}=0} \sigma_{A}\left(a+a^{\prime}\right)=\mathbf{D}$, while $\sigma_{B}(T a)=\eta \sigma_{B}(T a)=\frac{1}{2} \mathbf{D}$.

Notice also that (1.6) fails, in spite of the fact that $T$ is one-one, and that (2.5) fails, in spite of the fact that $T$ has dense range.

3. We have tried to suggest that the exponential spectrum of a Banach algebra element is in some sense dual to the singular spectrum. There are some defects in this, however: for example it is well known [3] that the singular spectrum obeys the spectral mapping theorem for polynomials:

$$
f \tau_{A}(a)=\tau_{A} f(a)
$$

whenever $a \in A$ and $f: \mathbf{C} \rightarrow \mathbf{C}$ is a polynomial. In contrast we have only

$$
\varepsilon_{A} f(a) \subseteq f \varepsilon_{A}(a)
$$

which follows from the fact that $\operatorname{Exp}(A)$ is closed under multiplication. A 
counterexample to equality can be found in Berberian [1, Example 3.3]. In another direction recall (2.4): the exponential spectrum behaves at least as well as the ordinary spectrum under arbitrary homomorphisms. In contrast we can say nothing about the singular spectrum, either in the same or in the opposite direction. This is clear from Example 1; the following two quotient examples were used by Smyth and West [4] in another context.

ExAmple 2. If $A=Q(D)$ and $J$ is the closed ideal generated by $z^{2}$, and if $a=z \in A$, then

$$
\tau_{A / J}(a+J) \nsubseteq \tau_{A}(a) .
$$

For the coset $a+J$ is nilpotent, giving $\sigma_{A / J}(a+J)=\{0\}$, hence 0 $\in \tau_{A / J}(a+J)$; on the other hand, 0 is excluded from $\tau_{A}(a)$ by the maximum modulus principle.

EXAMPLE 3. If $A=Q(\mathbf{D})$ and $J=\left\{a \in A: \partial \mathbf{D} \subseteq a^{-1}(0)\right\}$, and if $a=z$ $\in A$, then

$$
\bigcap_{a^{\prime}=0} \tau_{A}\left(a+a^{\prime}\right) \nsubseteq \tau_{A / J}(a+J) .
$$

This reduces to the corresponding inclusion for spectra. Since $A / J$ $\cong \mathcal{C}(\partial \mathbf{D}), 0$ is not in $\tau_{A / J}(a+J)$; to see that 0 must be in the left-hand side observe [4] that any function $a^{\prime} \in J$ for which $a+a^{\prime}$ is invertible in $A$ must induce a mapping from $\mathbf{D}$ to $\partial \mathbf{D}$ which is incompatible with Brouwer's fixed point theorem.

We can also observe (3.4) in the Calkin algebra of a Hilbert space $H$. Recall that this is $B=A / J$ where $A=\mathscr{B}(H)$ and $J=\mathscr{K}(H)$ is the ideal of compact operators. It is familiar [2, Corollary 5.30] that $A^{-1}=\operatorname{Exp}(A)$, so that the exponential spectrum of a bounded operator is just the ordinary spectrum. In the algebra $B$ the invertible elements $B^{-1}$ are [2, Theorem 5.17] the cosets of Fredholm operators, while the generalized exponentials $\operatorname{Exp}(A)$ are $[2$, Theorem 5.35] the cosets of Fredholm operators of index zero. Thus the spectrum of $a+J$ in $B$ is the Fredholm essential spectrum of $a \in A$, while the exponential spectrum of $a+J$ is the Weyl spectrum of $a$. If $T: A \rightarrow B$ is the natural mapping then (2.5) reduces to Schechter's theorem [1, Theorem 2.5]. Now (3.4) occurs whenever $a \in A$ is Fredholm of index $\neq 0$; also strict inequality in (3.2) can be obtained for $T a \in B$ by arranging that $a+1$ and $a$ -1 have index +1 and -1 respectively, and taking $f(z)=z^{2}-1$ [1, Example 3.3], [3].

\section{REFERENCES}

1. S. K. Berberian, The Weyl spectrum of an operator, Indiana Univ. Math. J. 20 (1970/71), 529-544. MR 43 \#5344. 
2. R. G. Douglas, Banach algebra techniques in operator theory, Academic Press, New York, 1972. MR 50 \# 14335.

3. B. Gramsch and D. C. Lay, Spectral mapping theorems for essential spectra, Math. Ann. 192 (1971), 17-32. MR 45 \# 936.

4. M. R. F. Smyth and T. T. West, The spectral radius formula in quotient algebras, Math. Z. 145 (1975), 157-161.

5. J. L. Taylor, Banach algebras and topology, Algebras in Analysis, Academic Press, New York, 1975.

Department of Mathematics, University College, Cork, Ireland 